\title{
Aspectos psicosociales y gestión de la evaluación en secundarias de alto y bajo logro
}

\section{Psychosocial aspects and management of evaluation in secondary high and low achievement}

Investigación

Alicia Rivera Morales

Universidad Pedagógica Nacional-México alirimo@hotmail.com

Recibido: 16 de octubre de 2015 / Aceptado: 13 de enero de 2017

\section{Resumen}

En este artículo se presenta un segmento de un estudio de corte mixto más amplio, se recupera una parte del análisis cualitativo en torno a los aspectos psicosociales y la gestión de la evaluación, es resultado de la aplicación de entrevistas semiestructuradas y grupos de enfoque a ochenta directivos de escuelas secundarias de alto y bajo logro educativo ubicadas en cuatro entidades de México: Sonora, Durango, DF y Oaxaca. Los hallazgos se exponen a partir de categorías que consideran el significado, las creencias y expectativas acerca de la evaluación, así como la gestión de la evaluación de los directivos de las escuelas estudiadas. Los casos polarizados permiten, en sus contrastes, identificar procesos y elementos que marcan diferencias o coincidencias entre ellos. De esta manera se encontró que los directivos de las escuelas de bajo logro esperan: cambiar la actitud de los docentes frente a la evaluación, que los supervisores controlen a los docentes y el apoyo externo (especialistas) que les indiquen como evaluar. Por otra parte, se reafirma la idea de que una escuela con alto logro, es una organización en la que el director propicia la participación, tiene altas expectativas de alumnos y docentes, su sistema de gestión y toma de decisiones permite alcanzar los objetivos establecidos y propone acciones de evaluación que buscan la mejora continua.
Descriptores: Aspectos psicosociales, gestión escolar, evaluación, escuelas secundarias, alto y bajo logro educativo.

\section{Abstract}

This article describes a segment of a broader study of mixed cut occurs. In this space only part of the qualitative analysis is recovered around the psychosocial aspects that influence the management of the evaluation results from the application of semi-structured interviews and focus groups with 80 senior secondary schools with high and low achievement located in four states of Mexico: Sonora, Durango, Mexico City and Oaxaca. The findings are presented from categories they consider the meaning, beliefs and expectations about evaluation and how these aspects influence the actions of the directors of the schools studied. Polarized cases allow, in their contrasts, identify processes and factors that mark the differences or similarities between them. Thus it was found that school principals low achievement hope to change the attitude of teachers towards the assessment, expect supervisors to monitor teachers and external support (specialists) that indicate how to evaluate them. Moreover, the idea that a school with high achievement is an organization that promotes the participation director, has high expectations of students and teachers, its management

Forma sugerida de citar: Rivera Morales, Alicia (2017). Aspectos psicosociales y gestión de la evaluación en secundarias de alto y bajo logro. Alteridad, 12(1), pp. 92-103. 
system and decision-making can achieve the objectives set, and reasserts Assessment proposes actions that seek continuous improvement.

\section{Introducción}

La calidad de la educación es una preocupación especial en la agenda de las políticas educativas. En México, se impulsa una vez que se consolida la masificación de la oferta de la educación básica. Esta preocupación ha tenido como uno de sus resultados tangibles la motivación por hacerla visible y medible. De manera que, no sólo se trata de desarrollar sistemas de medición a nivel nacional, comparando por estratos, regiones o niveles de desarrollo. Los procesos de evaluación impuestos desde la política en los últimos años en las escuelas, demandan rediseñar la función directiva, los supervisores y directores, quienes reconstruyen su papel en la escuela, simplificando radicalmente sus tareas administrativas y apoyando su condición de líderes académicos.

En este trabajo, que forma parte de un estudio más amplio, se pretende ilustrar de qué manera influyen algunos aspectos psicosociales, tales como el significado, expectativas y creencias en la gestión de la evaluación de ochenta directivos de escuelas secundarias públicas de los Estados de Sonora, Durango, Oaxaca y Distrito Federal, seleccionando para ello casos polarizados por niveles de logro educativo (20 de alto nivel y 20 de bajo logro). El artículo está constituido por cuatro apartados: 1) fundamento teórico; 2) método; 3) hallazgos y 4) conclusiones.

\section{Fundamento teórico}

Uno de los aspectos que han impulsado el renacimiento de la escuela como foco de atención de investigaciones, lo constituye el movimiento de administración basado en escuelas y de las escuelas efectivas; movimiento que en Latinoamérica coincide con los procesos de descentralización educativa (Slavin, citado en Loera, Cázares,
Keywords: Psychosocial aspects, management, evaluation, secondary, high schools and low educational attainment.

García, González, Hernández, \& G. de Lozano, 2003). La dimensión organizacional de la política educativa considera a sus actores básicos (docentes, directivos, alumnos y padres de familia) en su esfuerzo por constituir, en el espacio institucional de la escuela, una comunidad de aprendizaje efectivo y significativo (Rivera y Rivera, 2006).

Las investigaciones realizadas han sido numerosas, centradas en escuelas eficaces y tienen su origen en el estudio de Coleman (1990) sobre la igualdad de oportunidades en educación. Estudios clásicos en el campo de las escuelas eficaces, se puede citar: el de Rutter et al. (1979) realizado en escuelas del nivel secundaria estableció siete factores vinculados con la eficacia de las escuelas y el de Mortimore (1991) señaló que existen condiciones propias de la escuela tales como el tamaño, el ambiente y a la estabilidad de los profesores que favorecen su mejoramiento. Los factores señalados por Sammons et al. (1995) ofrecen una imagen que perfila las características de las escuelas eficaces, entre otras se encuentran: liderazgo, visión y metas compartidas, expectativas elevadas, entre otras. Similares conclusiones se encuentran en diversas investigaciones en América Latina. Por mencionar, Arancibia (1992), Espinoza y cols. (1995), Zárate (1992), Servat, (1995) y Alvariño y Vizcarra (1999) resaltan como factores claves de las escuelas efectivas, el sentido de misión compartido por directivos y profesores. En tal sentido son relevantes los trabajos de Teddlie \& Stringfield (1993), Reynolds (1996) y Stoll \& Fink, (1999). A la luz de estos estudios aparece con claridad que hay ciertos factores adicionales relevantes para explicar la ineficacia o pérdida de eficacia de las escuelas. Por ejemplo: ausencia de liderazgo (directores con bajas expectativas, poco comprometidos). Dentro de los investigadores que pretenden aproximar ambos paradigmas se encuentran: Hargreaves (1994) Hopkins (1994); 
Ainscow, Hopkins, Soutworth, \& West (1994); Stoll y Fink (1999).

Para la evaluación de las escuelas se han inspirado principalmente en el estudio de Stufflebeam y Shinkfield (1985), Stake (1967), Provus (1971) y en los avances realizados en el campo de la metodología para la medición del valor agregado, específicamente el modelo lineal jerárquico (HLM, por sus siglas en inglés Hierarchical Linear Model), como lo describen Bryk y Raudenbush (1992), Goldstein (1988). La evaluación de las escuelas se concentra en los resultados de los alumnos, medidos por test estandarizados, como si éstos fuesen los únicos resultados válidos de la actividad del estudiante (Gardner, 1995). Loera, Cázares, García, González, Hernández, \& G. de Lozano (2003), incluyen en su estudio: consenso sobre los objetivos de la escuela, liderazgo, oportunidades de aprendizaje, clima escolar y la interacción entre los profesores. Dichos indicadores de resultado proporcionan información sobre definiciones de "buenas escuelas" y "buenas prácticas de gestión" o escuelas de alto nivel de logro.

Por su parte, Horn y Murillo en un estudio multinivel discuten la incidencia de la dirección escolar sobre el compromiso de los docentes (Horn y Murillo, 2016); en otro artículo se cuestionan qué tareas de los directores y directoras escolares son las que más inciden en el aprendizaje de los estudiantes (Murillo, y Hernández-Castilla, 2015); Murillo y Krichesky (2015) ponen énfasis en la mejora de la escuela a partir de las lecciones aprendidas. Estos estudios dieron pie a problematizar sobre la cultura escolar desde una perspectiva psicosocial, se trata de un acercamiento a las representaciones, creencias, expectativas, valores, poder, elementos subjetivos inherentes en toda acción humana cuando se llevan a cabo procesos evaluativos. Para el caso que se ocupa, se pretende responder las siguientes interrogantes ¿De qué manera se representan los directivos la evaluación?, ¿Cuáles son sus expectativas y creencias acerca de la evaluación educativa?, ¿Cómo gestionan dicha evaluación en sus escuelas, qué acciones realizan? ¿Existen diferencias en los elementos y la gestión de la evaluación en escuelas secundarias de alto y bajo logro?

\section{Método}

La investigación amplia es de corte mixto con perspectivas cuanti y cualitativa Hernández, Fernández, \& Baptista (2008). Es de tipo comparativo ya que se han manipulado los escenarios: fueron seleccionadas diez escuelas secundarias en cuatro entidades, cinco de alto y cinco de bajo logro, con base en los resultados de la Evaluación Nacional del Logro Educativo en Centros Escolares (ENLACE). Para el caso de Oaxaca (que no participó en esa prueba) fueron seleccionadas de acuerdo a la perspectiva de las autoridades educativas estatales. En total fueron cuarenta escuelas. Se utilizó un método cualitativo, comparativo, de estudios de casos. La consideración de las escuelas como estudios de caso, tiene como fundamento abordar la manera como los directivos se representan o le dan significado a la evaluación, sus creencias, expectativas y de qué manera la llevan a cabo en la institución escolar que dirigen. En este segmento de la investigación general sólo se consideran los 80 directivos, a quienes se les aplicaron entrevistas semiestructurada y grupo de enfoque con directivos de AL y BL por cada entidad si era posible o de lo contrario se utilizó la entrevista semiestructurada.

\section{Análisis y resultados}

El análisis cuantitativo se llevó a cabo con el programa SPSS, mientras que el cualitativo, que es el que se presenta en este documento, se hizo con base a categorías preestablecidas y subcategorías que emergieron de los hallazgos.

En este caso, se consideran cuatro dimensiones: significado, expectativas, creencias $\mathrm{y}$, acciones de los directivos en torno a la evaluación.

Significado.- Incluye el concepto que le otorgan los directores a la evaluación. El significado es un proceso cognoscitivo de atribución a 
experiencias o a conocimientos personales, de un sentido suplementario que se vuelve preponderante en lo vivido por el sujeto (Psycholobiology Glossaire, 2007). Es el resultado del cúmulo de conocimientos del individuo, constituye su mirada de la realidad vivida, como la única lectura verdadera para él y que puede comprenderse por otros inidividuos. Son productos sociales que tienen una historia (Berger y Luckman, 1968).
Los directivos de escuelas de bajo logro, tienen una concepción ortodoxa de la evaluación, así lo manifiestan:

... la evaluación, es un acto en el proceso para revisar qué tanto se están logrando los objetivos planteados $(\mathrm{DB})$.

Por otro lado, los directivos de las escuelas de alto logro, señalan a la evaluación como un proceso continuo: (Ver figura 1):

\section{Figura 1: Significado de la evaluación}

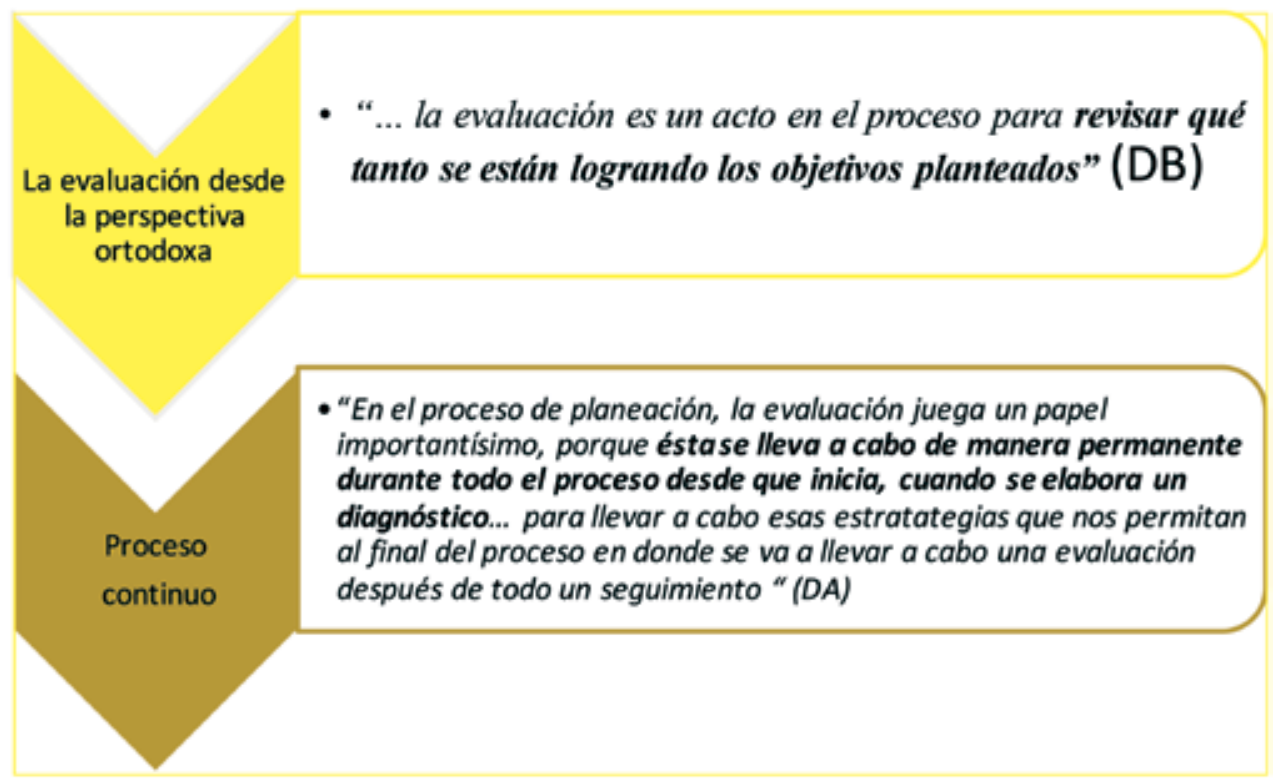

\section{Fuente: Elaboración propia}

Es interesante observar que, tanto en la representación como en el significado de la evaluación, los directivos de las escuelas de $\mathrm{AL}$ y $\mathrm{BL}$ existen ciertas coincidencias en cuanto señalan que la evaluación permite revisar los objetivos, rendir cuentas y realizar diagnósticos en sus escuelas. Este estudio, de acuerdo con Berger y Luckman (1968), refrenda que el conocimiento del directivo, su mirada de la práctica evaluativa y lo que hace, le da significado para él y los otros agentes educativos (docentes y estudiantes). Por tanto el significado de la evaluación de los directores de escuelas de AL y BL, es resultado de un proceso social y cultural compartido en la organización escolar. No es el caso de las acciones que desarrollan para llevar a cabo la evaluación, lo que se contrapone con la idea de que la concepción fundamenta la acción.

Expectativas.- incluye lo que se espera lograr a través de la evaluación, a partir de una proposición en la que confían y que los lleva a actuar. Es decir, una profecía que se autocumple, una suposición o predicción que, por la sola razón de haberse hecho, convierte en realidad el suceso supuesto, esperado o profetizado y de esta manera confirma su propia exactitud (Watzlawick, 1993, citado en Santos, 
2001). En este aspecto, directores y directoras de escuelas de AL esperan de y con la evaluación: generar ambientes adecuados para llevarla a cabo, asì como generar una cultura evaluativa individual y colectica. Plantean que es importante recuperar los aciertos y los errores del Sistema Educativo, así como revisar si sus escuelas tienen demanda o si los alumnos no asisten, para aprender y mejorar.

...dentro de los procesos de evaluación nos damos cuenta si la escuela funciona por la demanda que tiene, si los padres siguen mandando a sus hijos ahí,. Si los alumnos ya no van a esa escuela quiere decir que algo anda mal... (DEA)

Estos directivos señalan que la evaluación debe ser real y no maquillada, proponen herramientas que les permiten avanzar en la obtención de mejores resultados con el compromiso de todos porque "el director no lo puede hacer todo" (Ver figura 2).

Figura 2: Expectativa directores alto logro

Genera ambientes adecuados para la evaluación

“... Para que haya una buena evaluación se tiene que estar siempre creando un clima bueno entre el personal docente, intendente, secretarias "

Generar una cultura evaluativa individual y colectiva

“ ... hacer visitas a los docentes, podemos ir enriqueciendo, ir generando una mueva cultura de la evaluación..."

Reconocer las debilidades para avanzar y cambiar

"Nuestro sistema educativo ... hay situaciones buenas, situaciones malas... se tiene que ver como cambiar lo negativo, las fallas, los errores... de los erroresse tiene que aprender y se tiene que mejorar... dentro de los procesos de evaluación nos damos cuenta si la escuela funciona por la demanda que tiene, si los padres siguen mandando a sus hijos ahi. Si los alumnos ya no van a esa escuela quiere decir que algo anda mal..."

La evaluación sea real

"Cuando las evaluaciones no se maquillen, que realmente sean reales en cuanto al avance del educando, per que tiene como propósito el reflejarnos una realidad para poder seguir avanzando, seguir creciendo y sobre todo con el compromiso de toda la cominidad, porque el director no lo puede hacer todo..."

\section{Fuente: Elaboración propia}

Por otro lado, los directivos de las escuelas de BL esperan de y con la evaluación que los supervisores monitoreen la evaluación en el aula y de esta manera evitar que el docente improvise sus actividades de planeación, así como que se actualicen a través de cursos, talleres o seminarios en temas relacionados con la evaluación. Esperan, además que el alumno adquiera las habilidades para contestar un examen (Ver figura 3). 
Que los supervisores monitoreen la evaluación. "Para que una escuela brinde servicios de calidad tiene que ocurrir un fenómeno el maestro tiene que planear su actividad no puede llegar a improvisar nada más y esa planeación tiene que ser monitoreada por un administrador educativo..."

Que los profesores se preparen en evaluación "...que realmente se llevara a cabo un curso, seminario o taller donde se viera exactamente qué es la evaluación y qué instrumentos puede utilizar el maestro para evaluar..."

Que el alumno desarrolle habilidades para responder el examen. "El alumno tiene que aprender a desarvollar todos esos tipos de habilidades de pensamiento... también las habilidades que le podrán avudar realmente a contestar un examen"

\section{Fuente: Elaboración propia}

Los directores de las escuelas de BL esperan mayor control por parte de los supervisores a los docentes, que éstos se actualicen en evaluación a fin de mejorar sus prácticas evaluativas y los estudiantes aprendan a responder exámenes.

Tal proposición en la que confían los lleva a actuar a unos y otros directivos. Es decir, la profecía se cumple, una suposición negativa: "el docente no sabe, no planea, requiere de actualización"; o positiva: "un buen clima genera una buena evaluación, de los errores se aprende" son predicciones que, por la sola razón de haberse formulado, se convierten en realidad. La información recabada sostiene que la expectativa, el suceso esperado o profetizado se confirma en la realidad. En este aspecto si se encontraron diferencias significativas entre los directivos de AL y BL, mientras los primeros tienen altas expectativas, realizan acciones para conseguir lo que se proponen a través del apoyo que brindan a sus maestros y alumnos, trabajan de manera colaborativa, mientras que los segundos tienden a formular profecías negativas de los actores que liderean y esperan que el sistema, los otros, el contexto cambien.

Creencias.- De acuerdo con Villoro (2004) las creencias significa tener algo por verdadero pero sin estar seguro de ello, ni contar con pruebas suficientes. Es un acto de una cualidad específica que ocurre en la mente de un sujeto y sólo podremos conocer el pensamiento de alguien a través de los actos de ese sujeto, en su relación con el mundo que lo rodea (Villoro, 2004). Una creencia es una afirmación que consideramos verdadera consciente o inconscientemente, afecta a la percepción que tenemos de nosotros mismos, de los demás y del mundo en general. Es todo aquello que creemos verdadero e importante para nosotros.

Los directivos de escuelas de AL creen que el reto es saber si el alumno adquirió todos los saberes, conceptual, procedimental y actitudinal, creen que pueden incidir en las prácticas educativas pero no en la realidad que cambia día con día; que para gestionar la evaluación es importante partir de la realidad, determinar las condiciones en las que se encuentran y luego establecer compromisos entre 
la comunidad para crecer de manera eficiente. Añaden que para lograrlo las evaluaciones deben ser reales, de lo contrario queda en un simple informe y todo continúa igual. Para poder cambiar es necesario aceptar que existe un problema y luego establecer compromisos entre compañeros y comunidad para seguir.

Por otra parte señalan que la discriminación y calificación entre maestros es más común de lo que se cree, por ser maestros de carrera o de otras especialidades, sin embargo, señalan que cada individuo tiene competencias diferentes y que el número puede o no corresponder a valorar dichas competencias, la inteligencia o el aspecto emocional, esta situación es igual con alumnos, maestros y con los directivos (Ver Tabla 1).

Tabla 1: Creencias de los directivos

\section{Alto}

\begin{abstract}
El reto es lograr que el maestro pueda registrar los aprendizajes"...ese es el reto, de qué manera el maestro puede registrar que el alumno llegó a adquirir esa habilidad, ese conocimiento, esa actínd ...cómo medimos ese aprendizaje, cómo nos damos cuenta que el ahumno lo alcanzo..."(DA)
\end{abstract}

Para gestionar la evaluación es importante partir de la realidad "el problema de la escuela no se ha adaptado a esta mieva realidad..."

Existe discriminación entre los docentes. "La mayoria, sean ,maestros de carrera, sean de potras carrer as está discriminando y sancionando - tú si eres bueno, tú eres mediano y tú eres malo... igual es con los maestros, igual es con los directivos-..."

Participación de todos los actores en la evaluación

Creen en la formación de los docentes

\section{Bajo}

\section{Si se evalúa el desempeño docente mejorarán las cosas}

Existe incongruencia entre los niveles evaluativos. "Evaluación no coincide con la forma de evahuar otros miveles...aqui en la evahación contimia, se evahian una serie de aspectos lo cual dan un resullado final, y cuando el alumno egresa de la secundaria tiene que enfrentarse a un examen de conocimiento totalmente al proceso de evaluación que se lleva en la secandaria y por eso muchas fracasan" (DB)

Reproduce formas de evaluación "el profesor tiende a repetir todos los procesos como fue formado, el maestro evaluia como lo evaluaron... estos exquemas los tiene bien establecidos y es difficil modificarlos, hay una resistencia al cambio..."

Carencias de formación pedagógica "...el $80 \%$ de los que componemos la planta docente en secundaria no son normalistas, huego entonces carecen de elementos técnicas pedagógicas-didácticas.

\section{Fuente: Elaboración propia}

Los directores y directoras de las escuelas de AL poseen creencias más positivas e insisten en la necesidad de promover la participación de todos los miembros de la comunidad, incluyendo a los padres de familia.

Mientras que los directivos de las escuelas de BL creen necesario evaluar al maestro, al alumno, al padre de familia, la deserción, eficiencia terminal. Afirman que el docente cree que está bien y cuenta con conocimientos pero en realidad debe actualizarse, prepararse de manera constante. Refieren que existe incongruencia entre los procesos evaluativos que se llevan a cabo en las escuelas secundarias y a los que tienen que sujetarse los estudiantes cuando egresan de las mismas (examen único de ingreso al nivel medio superior), razón por la cual muchos alumnos fracasan. Creen que el responsable de esta situación son los docentes quienes realizan una evaluación de corte cualitativo y no cuantitativo, y éste es el solicita- 
do al estudiante para su ingreso al nivel medio superior. Señalan que el docente evalúa como lo evaluaron. Consideran que por ser la mayoría docentes egresados de la universidad carecen de elementos técnico-pedagógicos-didácticos.

Estos esquemas mentales son difíciles de modificar y existe resistencia al cambio. Los directivos de las escuelas de BL creen que la evaluación es un problema ontológico, que los docentes necesitan ser controlados y para ello aplican una serie de instrumentos para medir la deserción, la eficiencia terminal; creen que el aprendizaje es el principal objeto de evaluación; que existe incongruencia entre los procesos evaluativos de la escuela secundaria y los solicitados externamente; que el docente es el responsable de que sus egresados no se incorporen al nivel medio superior debido a que reproducen procedimientos de evaluación que ellos vivieron como alumnos y carecen de formación en estrategias pedagógicas y de evaluación.

Por otro lado, los directores de escuelas de alto logro creen en la autoevaluación de la gestión, en trabajar con los profesores y aprender con los alumnos, creen que su ámbito de incidencia es acotado; que sólo si se parte de la realidad y con evaluaciones objetivas se puede crecer; evaluar continuamente para que haya éxito en la tarea; que para cambiar de actitudes es necesario evaluar las habilidades, actitudes y aptitudes y, que las reformas curriculares se realizan sin que medie una evaluación de las mismas y creen en la participación de todos los actores en los procesos evaluativos.

Las creencias de los directivos, afirmaciones que consideran verdaderas consciente o inconscientemente, afectan la percepción que tienen de ellos mismo, de los demás y del mundo en general, tal afirmación los lleva a actuar. Sus actos como concreción de su pensamiento los hace diferentes: los de AL emprenden acciones para alcanzar buenos logros; mientras que la creencia de los directivos de BL los paraliza y responsabilizan al entorno social, económico, político de su nivel de logro.

Acciones o gestión de la evaluación.- La acción humana dentro de las organizaciones esco- lares para llevar a cabo la evaluación educativa. O dicho de otra manera, la gestión es la capacidad de articular los recursos de que se disponen de manera de lograr lo que se desea (Casassus, 2000).

Los directivos de escuelas de AL asumen lo difícil que puede ser, desde su ámbito, el poder cambiar prácticas históricas que propicien la mejora del quehacer educativo. Estos actores educativos, en sus márgenes de acción, llevan a cabo su gestión, poniendo en marcha una serie de estrategias y para ello recuperan su realidad para tomar decisiones..." mi visión es a pesar de todo lo que existe, como existe y como está, atender la problemática de mi institución que es lo que más me preocupa" (DA).

Las sugerencias que plantean los directivos de escuelas de alto logro son variadas y se refieren a lo siguiente: brindan apoyo, los recursos materiales necesarios al profesorado para que desarrolle su labor.

...no le vamos a decir qué haga y cómo le haga es problema de él, pero si brindarle todo ese apoyo, todos los recursos materiales posibles, todos los recursos tecnológicos, el uso de la computadora y apoyar a los compañeros maestros (DA).

También es importante hacer un seguimiento de lo que hace el docente en su aula, en la evaluación.

...si un maestro de matemáticas pasa todos sus alumnos con diez pues hay que irlo a ver, ver que hace en la zona, el sector, hay que darse cuenta como lo está haciendo...y si un maestro saca puro cinco, pues también hay que irlo a ver, que está haciendo, para poder apoyar ese proceso (DA).

Acompañan al docente en su trabajo pedagógico y reiteran la relevancia del trabajo colegiado para establecer un diálogo sobre sus logros y rezagos a fin de comprenderlos y poder mejorar lo que se hace en el terreno de la evaluación. "Como el mecánico que no está actualizado ya no tendrá trabajo y el maestro, ¿qué pasa con él?” 
Señalan que es importante crear un clima que favorezca los procesos de evaluación en la escuela, tienen claro que el estudiante es su prioridad a quien tienen que darle su vida, su trabajo para prepararlo para la vida y garantizar que los padres de familia sigan confiando en la escuela y la recomienden como una buena institución educativa.

...para que haya una buena evaluación se tiene que estar siempre creando un clima bueno entre el personal docente, intendente, secretarias, todo el personal de apoyo para que todos nos centremos ... es el clima que se tiene que crear constantemente si hay fallas inmediatamente corregirlo (DA).

Los directores de AL, insisten en la necesidad de establecer un clima de confianza donde los alumnos puedan externar sus sentimientos y problemas familiares a fin de facilitar un mejor aprendizaje y un ambiente propicio para la evaluación.

...crear el clima, le decíamos a los padres de familia en las primeras reuniones no nos manden a sus hijos todos traumados y temerosos, cansados porque antes de salir de casa tuvieron ustedes un pleito fuerte delante de ellos y el pobre niño no viene aquí aprender porque esta rumiando el problema que vivió en casa, vamos agarrar esos 50 minutos que tenemos en clase ... me da tal maestro que me cae a todo dar y ahí aprendo, pues crear ese clima de tranquilidad un ambiente sano, un ambiente bueno, yo creo que eso nos va a dar la mejor evaluación (DA).

Por otra parte, se manifiesta, en los comentarios que el significado de la evaluación se ha modificado y ha pasado de ser una referencia a la valoración de los aprendizajes a un concepto aplicado a otros objetos del sistema educativo, para ello proponen realizar un ejercicio de autoevaluación de su gestión, considerando el clima organizacional y su liderazgo efectivo (Ver figura 5).

Esto no lo es todo porque también tenemos que valorar, el clima organizacional, que tipo de liderazgo que el director está ejerciendo, qué tipo de comunicación se está dando (DA).

Los directivos en escuelas de bajo logro reflexionan sobre la práctica directiva en relación con la evaluación, un ejercicio que los lleva a proponer procedimientos de evaluación elaborado por su cuerpo docente a fin de mejorar los procesos evaluativos en sus escuelas (Ver figura 5).

Por otra parte, los directivos de las escuelas de BL insisten que el problema está en los maestros, entonces cambiando ellos, de actitud y estén convencidos podrán llevar a cabo la evaluación de manera distinta, pero ello sólo se podrá lograr a través de seminarios, talleres o cursos donde se sensibilice o casi se le obligue a comprometerse a ser un profesional de la evaluación.

...pues buscar sensibilizar al maestro y que evalúe verdaderamente lo que tiene que evaluar lo que establece en los planes y programas de estudio, mientras que el maestro no esté plenamente convencido de qué debe evaluar ... no vamos a lograr ese cambio, que le podrán ayudar realmente a contestar un examen; ¿cómo podemos hacerlo? insisto, diseñando un seminario, taller, curso donde verdaderamente el profesor se le sensibilice, se le, casi, casi se le obligue a que verdaderamente se comprometa a ser un profesional en la evaluación (DB).

Solicitan que la solución sea propuesta por especialistas, del sistema educativo para resolver los procesos evaluativos en sus escuelas o bien les proporcionen instructivos para llevarlos a cabo.

...la propuestas buscar apoyos externos o apoyos de las supervisiones, que sería una de las funciones de la supervisión, apoyar los procesos de la escuela...(DB) "Pues, para mí sería que editaran algún instructivo, libro, folleto, donde si se estableciera la forma de evaluar por un investigador o hacer una compilación de todos los investigadores y que fuera obligatorio en el sistema de secundaria, todas, que llevara un modelo de evaluar (DEB). 
Figura 5: Acciones de los directores

\section{ALTO}

\begin{tabular}{|l|}
\hline $\begin{array}{l}\text { Brindan apoyo y seguimiento al } \\
\text { docente }\end{array}$ \\
$\begin{array}{l}\text { Acompañan al docente en su trabajo } \\
\text { técnico-pedagógico }\end{array}$ \\
Generan nuevos ambientes para la \\
evaluación
\end{tabular}

\section{Fuente: Elaboración propia}

Los directivos de escuelas de BL centran su propuesta de mejora de los procesos evaluativos en la necesidad de cambiar la actitud de los docentes frente a los procesos evaluativos; de unificar criterios para llevarlos a cabo y consideran que sería importante que se les otorgue un instructivo, folleto donde varios investigadores les digan cómo evaluar.

Los directivos de las escuelas de AL reiteran la relevancia del trabajo colegiado para establecer un diálogo sobre sus logros y rezagos a fin de comprenderlos y poder mejorar lo que se hace en el terreno de la evaluación. Intentan propiciar un ambiente que favorezca los procesos de evaluación en la escuela cuyo beneficiario es el estudiante, insisten en la necesidad de establecer un clima de confianza donde los alumnos puedan externar sus sentimientos y problemas familiares a fin de facilitar un mejor aprendizaje. La gestión de la evaluación en escuelas de AL y BL presenta diferencias importantes (Ver figura 5). Estos hallazgos coinciden con resultados de estudios de prácticas de gestión exitosas de directivos de escuelas de altos niveles de logro educativo.

\section{BAJO}

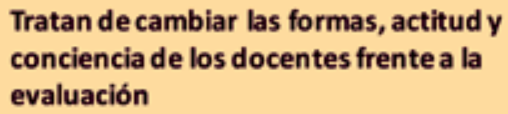

Tratan de cambiar las formas, actitud y conciencia de los docentes frente a la evaluación

\section{Tratan de unificar criterios en} reuniones colegiadas

Buscan apoyo externo (supervisores,

especialistas

\section{Conclusiones}

La representación y el significado de evaluación de los directivos de las escuelas estudiadas, real o imaginaria, implícitamente coinciden, aunque en la acción los de AL muestran una representación distinta a la concepción ortodoxa de la evaluación. Este estudio refrenda que el conocimiento del directivo, su mirada de la práctica evaluativa y lo que hace, le da significado para él y los otros agentes educativos. Tal significado de la evaluación es resultado de un proceso social y cultural compartido en la organización escolar.

En relación con las expectativas, las de los directivos de escuelas de AL son altas; mientras que las de los de las escuelas de BL tienden a enunciar profecías de autocumplimiento dirigidas a los estudiantes y a ellos mismos, anuncian fracasos de tal manera que el fracaso acaba sucediendo y responsabilizan a los docentes del mismo.

Tal proposición en la que confían los lleva a actuar a unos y otros directivos. Los resultados de este trabajo sostiene que la expectativa, el suceso esperado o profetizado se confirma en la realidad. 
En este aspecto si se encontraron diferencias significativas entre los directivos de AL y BL.

En cuanto a las creencias, se presentan pensamientos dicotómicos: descalificación, etiquetación o lo expresión del deber ser. En este componente se manifiestan diferencias significativas entre los equipos directivos de los dos tipos de escuelas: los de alto logro ven experiencias más positivas mientras que los de bajo se van al otro extremo. Tales afirmaciones, que los directivos consideran verdaderas consciente o inconscientemente, afectan la percepción que tienen de ellos mismo, de los demás y del mundo en general y los lleva a actuar de manera diferente: los de AL emprenden acciones para sostener su nivel de logro; los de BL creen que si el entorno cambia entonces se podrá actuar de otra forma.

Se puede afirmar que, la acción humana dentro de las instituciones marca diferencias entre las escuelas de $\mathrm{AL}$ y $\mathrm{BL}$, en tanto que está generada por las expectativas y creencias de los directivos, en este caso. Al igual que Sammons et al. (1995), quien ofrece una imagen que perfila las características de las escuelas eficaces: liderazgo, visión y metas compartidas, expectativas elevadas, entre otras. Estos hallazgos coinciden con resultados de estudios realizados por Loera (2003) sobre prácticas de gestión exitosas de directivos de escuelas de altos niveles de logro educativo.

Los directores de las escuelas de alto logro reflexionan sobre su práctica de gestión, son más autocríticos y se incluyen dentro de las propuestas de mejora de los procesos evaluativos. Tienen una mirada positiva de sus docentes y son más propositivos. Los directivos de bajo logro responsabilizan el bajo aprovechamiento a los docentes, al sistema educativo, a los contextos, etc. y proponen que los docentes cambien sus procedimientos de evaluación para elevar los estándares solicitados, el riesgo en estas escuelas lo constituye el tener como foco de atención desarrollar mecanismos de evaluación que les permitan elevar su nivel de logro y no los aprendizajes de sus estudiantes.

\section{Referencias bibliográficas}

Ainscow, M., Hopkins, D., Soutworth, G., \& West, M. (1994). Creating the conditions for school improvement. En: A handbook of Staff Development activities. Londres: Roudledge.

Alvariño, C., \& Vizcarra, R. (1999). Gestión para la innovación en educación: desafíos para las escuelas particulares subvencionadas. En: P. Cariola, \& J. Vargas, Educación particular subvencionada. Santiago de Chile: CONACEP.

Arancibia, V. (1992). Efectividad escolar: Un análisis comparado. Estudios Públicos, 47, 101-125.

Berger P. \& Luckmann T. (1968). La construcción social de la realidad. Madrid: Amorrortu Editores.

Bryk, A. S., \& Raudenbush, S. W. (1992). Hierarchical Linear Models: applications and data analysis methods. California: Sage.

Casassus, J. (2000). Problemas de la gestión educativa en América Latina. UNESCO.

Coleman, J. (1990). Equality en Achievement in Education. Boulder, CO: Westview Press.

Espinoza, S., \& cols, y. (1995). Escuelas de calidad y sus procesos organizacionales: un cambio hacia el mejoramiento de la educación. Tesis de Licenciatura no publicada. Santiago de Chile: Pontificia Universidad Católica de Chile.

Gardner, H. (1995). Inteligencias múltiples. España: Paidós.

Goldstein, B. (1988). In Search of Survival: The Education and Integration of Among Refuges Girls. Journal of Ethnic Studies, 16(2), 1-27.

Hargreaves, A. (1994). Changing teachers, changing Times. Teacher' Work and Culture in the Postmodern Age. Londres: Cassell.

Hernández, R., Fenández, C., \& Baptista, P. (2008). Metodología de la Investigación (4ta ed.). México: McGraw Hill.

Hopkins, R. (1994). Narrative schooling: experiential learning and the transformation of American education. New York: Teachers collage.

Horn, A., \& Murillo, F.J. (2016). Incidencia de la dirección escolar sobre el compromiso de los docentes. Un estudio multinivel. Psicoperspectivas, 15(2). 
Loera, A., Cázares, Ó., García, E., González, M. d., Hernández, R., \& G. de Lozano, M. A. (Enero de 2003). El estado inicial de las escuelas primarias de las escuelas de calidad. Informe ejecutivo sobre los indicadores de la Línea de base. Obtenido de Heurística Educativa: http://basica.sep.gob.mx/pec/Documentos/ Evaluaciones\%20de\%20Heuristica/Reporte_ Ejecutivo.pdf

Murillo, F.J., \& Hernández-Castilla, R. (2015). Liderazgo para el aprendizaje: ¿Qué tareas de los directores y directoras escolares son las que más inciden en el aprendizaje de los estudiantes? RELIEVE, 21(1), art. 1.doi: 10.7203/ relieve.21.1.5015 (Scopus)

Murillo, F.J., \& Krichesky, G.J. (2015). Mejora de la Escuela: Medio siglo de lecciones aprendidas. REICE. Revista Iberoamericana sobre Calidad, Eficacia y Cambio en Educación, 13(1), 69-102.

Provus, M. (1971). Discrepancy Evaluation. California: McCutchan Publishing.

Reynolds, D. (1996). The problem of the ineffective school some evidence and some speculations. Londres: Cassel.

Rivera, A., \& Rivera, L. (2006). Organización, gestión y dirección de instituciones educativas. Reflexiones y propuestas. México: Mástextos UPN.
Rutter, M., Maughan, B., Mortimore, P., Ouston, J., \& Smith, A. (1979). Fifteen thousand hours: Secondary schools and their effects on children. Cambridge, MA: Harvard University Press.

Sammons, P., Hillma, J., \& Mortimore, P. (1995). Key Characteristics of Effective Schools: A Review of School Effectiveness Research. London: OFSTED/Institute of Education (University of London.

Santos, M. A. (2001). Teoría y práctica de la evaluación cualitativa de centros docentes. Madrid: Akal.

Servat, B. (1995). Gestión de recursos humanos en el centro escolar. Buenos Aires: Magisterio del Río de la Plata.

Stake, R. (1967). Evaluación comprensiva y evaluación basada en estándares. Barcelona: Graó.

Stoll, L., \& Fink, D. (1999). Para cambiar nuestras escuelas. Refundir la eficacia y la mejora. Barcelona: Octaedro.

Stufflebeam, D., \& Shinkfield, A. (1985). Evaluación sistemática: Guía teoría y práctica. Barcelona: Paidós.

Teddlie, C., \& Stringfield, S. (1993). Schools Make a Difference. New York: Teachers College Press.

Villoro, L., (2004) Creer, saber, conocer. Edit. Siglo XXI. México.

Zárate, G. (1992). Experiencias educacionales exitosas: Un análisis a base de testimonios. Estudios Públicos, 47, 127-158. 